\title{
Linking an Artery to the Circulation: Introducing a Quasi-Simultaneous Coupling Approach for Partitioned Systems in Hemodynamics
}

\author{
G. Rozema ${ }^{1}$, N.M. Maurits ${ }^{2}$ and A.E.P. Veldman ${ }^{1}$ \\ ${ }^{1}$ Institute of Mathematics and Computing Science, University of Groningen, the Netherlands \\ ${ }^{2}$ Department of Neurology, University Medical Center Groningen, the Netherlands
}

\begin{abstract}
When modeling complex systems such as the cardiovascular circulation one often needs to separate the problem into smaller subproblems because of the heterogeneous nature of the system and/or the modeling techniques. The application at hand is to link blood flow in an artery to that in the circulation. The artery of interest is modeled by a 3D CFD model that includes a model for the elastic wall to simulate fluid-structure interaction. This 3D arterial model is coupled to a $0 \mathrm{D}$ model (electric network) for the cardiovascular circulation. We show that the weak coupling method is numerically unstable for this application. Using a simple model problem, a theoretical stability analysis is performed which explains the instability of the simulation results. Additionally, we consider fluid-structure interaction in an elastic artery. Again stability analysis results in a theoretical (in)stability condition that is in good agreement with simulation results. As a solution for these stability problems the quasi-simultaneous method is presented. The big advantage over strongly coupled methods is its low computational cost.
\end{abstract}

Keywords - Partitioned systems, Hemodynamics, Stability analysis, Numerical simulation, Fluid-structure interaction.

\section{INTRODUCTION}

When modeling complex systems such as air flow around airplane wings or blood flow in the human circulatory system, often a modular approach is chosen: a detailed model is used when required and coarser models are applied whenever possible. In principle this is a costeffective approach. When solving the model, it is possible to treat all subsystems completely simultaneously (strong coupling), but this requires intensive merging of all submodels at algorithmic and software levels or the introduction of subiterations. This makes strong coupling a typically robust, but computationally expensive method. Alternatively, the subsystems can be coupled in a weak manner: the submodels are solved successively and the interface variables are exchanged only once per time step. While being cheap, stability is not guaranteed.

The application considered here is to link blood flow in an artery to that in the circulation, requiring separate submodels for blood flow, wall dynamics and circulation. Linking these submodels into a stable time-integration scheme is not straightforward. As it turns out, the weak method leads to an unstable integration scheme when wall elasticity is included. Here, a theoretical stability analysis is performed on a model problem in order to be able to predict such behavior. Furthermore, fluid-structure interaction in an elastic artery is investigated and theoretical and experimental stability bounds are shown to be close together.

As a solution for the stability problems, the quasisimultaneous method is presented. In this method a simple approximation of the arterial model - the interaction law - is solved simultaneously with the circulation model. In this case the interaction law is a OD approximation (RLC circuit) of the 3D CFD model. Because of the simplicity of the interaction law the quasi-simultaneous approach adds only little complexity and the computational effort per time step is hardly affected, without sacrificing stability.

\section{MATERIALS AND METHODS}

In this section partitioned systems are considered in a general setting and a theoretical stability analysis is performed on a model problem. The goal is to establish a theoretical background for weak coupling methods. The need arises because weak methods often turn out to be unstable and some basic insight into what causes these problems is needed in order to find a solution for it: the quasi-simultaneous method. The section is concluded with a simple theoretical consideration showing its usefulness.

\section{A. Partitioned systems: a model problem}

A partitioned system consists of two (or more) submodels relating the interface variables through which they are coupled. In a physical context, the interface variables generally are force $f$ and motion $x$. Consider the model problem consisting of a partitioned mass-springdamper system in figure 1 . For each of the masses $m_{1}$ and $m_{2}$ a separate equation is defined as follows:

$$
\begin{aligned}
& \text { system } 1:-f-\left(m_{1} \ddot{x}+\alpha_{1} \dot{x}+k_{1} x\right)=0, \\
& \text { system } 2: \quad f-\left(m_{2} \ddot{x}+\alpha_{2} \dot{x}+k_{2} x\right)=0,
\end{aligned}
$$






Fig. 1 Partitioned mass-spring-damper system

where $f$ represents the force between the two subsystems (which could easily be eliminated, but then the partitioned character is lost) and $x$ is the displacement of the system. Note that the masses $m_{1}$ and $m_{2}$ are rigidly connected, expressing the constraint that $x$ and $f$ are the same for both subsystems (the latter except for a minus sign). The system is written in shorthand notation as:

$$
\begin{aligned}
& \text { system } 1:-f-M_{1} x=0, \\
& \text { system 2: } \quad f-M_{2} x=0 .
\end{aligned}
$$

A weak coupling method uses a value of $f$ or $x$ at the old time level and the system is solved by substitution. For example, $x^{n}$ may be used by system 2 to compute $f^{n+1}$ after which $x^{n+1}$ is computed by system 1:

$$
\begin{aligned}
& \text { system 2: } f^{n+1}=M_{2} x^{n}, \\
& \text { system 1: } M_{1} x^{n+1}=-f^{n+1} .
\end{aligned}
$$

The weak method is a hierarchical solution method, with two possible iteration orders. Turning the iteration order around in (3) is of great effect on the stability of the method. In system (3) the force $f$ can be eliminated to yield $M_{1} x^{n+1}=-M_{2} x^{n}$. For stability the spectral radius of the iteration operator $-M_{1}^{-1} M_{2}$ must be smaller than one. The case with $\alpha_{i}=k_{i}=0$, i.e. $M_{1} x=m_{1} \ddot{x}$ and $M_{2} x=m_{2} \ddot{x}$ is highly illustrative. Then the amplification per time step is $-m_{2} / m_{1}$ and the weak approach is stable whenever $m_{2}<m_{1}\left(m_{2}>m_{1}\right.$ for the reversed order). The general case (1) is more complicated. Consider the following time discretization, with implicit spring and damper terms:

$$
M_{i} x^{n+1}=m_{i} \frac{x^{n+1}-2 x^{n}+x^{n-1}}{\delta t^{2}}+\alpha_{i} \frac{x^{n+1}-x^{n}}{\delta t}+k_{i} x^{n+1},
$$

and substitute in (3). Then the amplification per time step $\lambda$ satisfies the characteristic equation $a \lambda^{3}+b \lambda^{2}+c \lambda+d=0$ with $a, b, c$ and $d$ functions of the system parameters. For stability it is required that all roots lie within the unit disk: $|\lambda|<1$. With the transformation $\lambda=(z+1) /(z-1)$ the unit disk is transformed into the left half plane and the stability condition becomes $\mathfrak{R}(z)<1$. For a cubic equation $a z^{3}+b z^{2}+c z+d=0$ The Routh-Hurwitz theorem states that $\mathfrak{R}(z)<1$ if and only if $a, b, c$ and $d$ have the same sign and $b c>a d$. Using the positivity of the system parameters, it turns out that the coupled problem (3), (4) is theoretically stable if and only if

$$
\frac{4 \tilde{m}_{2}+2 \tilde{\alpha}_{2}+k_{2}}{4 \tilde{m}_{1}+2 \tilde{\alpha}_{1}+k_{1}}<1
$$

with $\tilde{m}_{i}=m_{i} / \delta t^{2}$ and $\tilde{\alpha}_{i}=\alpha_{i} / \delta t$. This condition is the generalization of $m_{2} / m_{1}<1$ for nonzero $\alpha_{i}$ and $k_{i}$. In section III the stability theory is applied to flow partitioning and fluid-structure interaction in hemodynamics.

\section{B. The quasi-simultaneous method}

Condition (5) illustrates that it is not always advisable to use a direct coupling method. For example when $m_{2}>m_{1}$ the method is numerically unstable for $\delta t \rightarrow 0$. For such cases we here present a solution that guarantees stability while maintaining the cost-effectiveness of the weak method: the quasi-simultaneous method.

In the quasi-simultaneous approach a simple approximation of one of the submodels is utilized. Suppose for example we have a simple version $I_{2}$ of the submodel $M_{2}$. The idea is then to solve $I_{2}$ simultaneously with $M_{1}$ which only becomes possible because of the simplicity of $I_{2}$, whereas solving $M_{1}$ and $M_{2}$ simultaneously would be too cumbersome. The difference between $M_{2}$ and its approximation $I_{2}$ is still treated in a weak manner. In short:

$$
\begin{aligned}
& \text { system 2: } f^{n+1}-I_{2} x^{n+1}=\left(M_{2}-I_{2}\right) x^{n}, \\
& \text { system 1: }-f^{n+1}-M_{1} x^{n+1}=0 .
\end{aligned}
$$

Note that $I_{2}=0$ corresponds to the weak method and $I_{2}=M_{2}$ to the fully simultaneous method. We here show that by using such an 'interaction law' stability can be achieved independent of system parameters as long as the approximation $I_{2}$ is 'good enough'.

To see how $I_{2}$ is helping to stabilize the system eliminate the force $f$ in the scheme (6) to obtain $x^{n+1}=-\left(M_{2}-I_{2}\right) /\left(M_{1}+I_{2}\right) x^{n}$. For stability again a spectral radius smaller than unity is required. To gain some insight on what this means suppose that $M_{1}$ and $M_{2}$ are simple scalar operators. Then the stability condition is simply $I_{2}>\left(M_{2}-M_{1}\right) / 2$. As known from the previous section the weak coupling method is unstable for $M_{2}>M_{1}$. Now however, we have the means of stabilizing such a system using the approximation $I_{2}$ that, according to the stability condition, only needs to be 'half as good' as $M_{2}$. In practice, simple models often satisfy this criterion and as such the quasi-simultaneous approach is quite useful. Below 
such an approach is implemented to couple a model of an elastic artery to a circulation model.

\section{RESUlts}

In this section two applications of the above stability analysis in hemodynamics are considered: flow partitioning and fluid-structure interaction (in an elastic artery). Stability conditions are derived and simulations are performed to demonstrate the usefulness of the quasi-simultaneous method and the predictive quality of the stability theory.

\section{A. Flow partitioning}

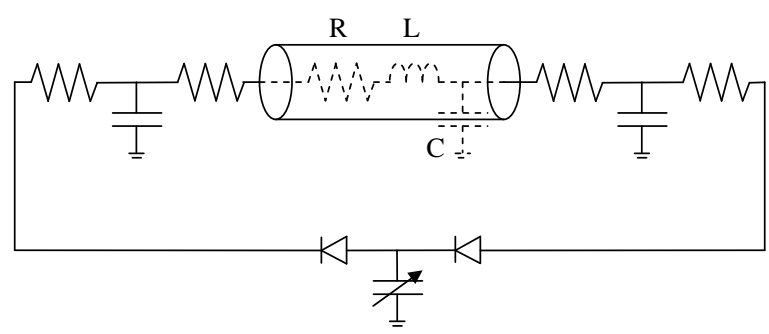

Fig. 2 3D CFD model of elastic artery coupled to circulation model

An electric network model of the circulation composed of two windkessel models and a simple ventricle model [1] is coupled to a 3D CFD model of an elastic artery to provide for boundary conditions (figure 2). The CFD model Comflo developed in-house employs a finite volume discretization of the Navier-Stokes equations [2,3]. Since the simulation is unstable (it blows up immediately), the stability of this weakly coupled system is investigated using the model problem in figure 3, consisting of an RLC circuit representing the $3 \mathrm{D}$ elastic artery and a three-element windkessel model.

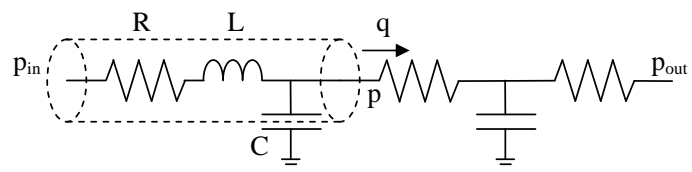

Fig. 3 Model problem: 3D elastic artery coupled to windkessel model

The pressure drop $p_{\text {in }}-p_{\text {out }}$ is prescribed. The equations for the RLC and windkessel subsystems can be expressed in terms of the interface variables $p$ (pressure) and $q$ (flow) as

$$
\begin{aligned}
& \text { RLC circuit : }-\left(p+a_{1} \dot{p}+b \ddot{p}\right)-\left(m_{1} \dot{q}+\alpha_{1} q\right)=-p_{\text {in }}, \\
& \text { windkessel : } \quad p+a_{2} \dot{p} \quad-\left(m_{2} \dot{q}+\alpha_{2} q\right)=p_{\text {out }} .
\end{aligned}
$$

Of particular interest is the capacitor $C$ which enters the equations in $a_{1}=R C$ and $b=L C$. The system (7) is obviously not of the form (1) since derivatives of both interface variables are involved. However, the theory in section II.A can be extended intuitively. When applying the weak coupling method with $q$ as input for the RLC circuit and $p$ for the windkessel model it can be shown that stability is achieved if and only if

$$
\frac{\left(1+2 \tilde{a}_{1}+4 \tilde{b}\right)\left(\alpha_{2}+2 \tilde{m}_{2}\right)}{\left(\alpha_{1}+2 \tilde{m}_{1}\right)\left(1+2 \tilde{a}_{2}\right)}<1,
$$

with $\tilde{a}_{i}=a_{i} / \delta t, \quad \tilde{m}_{i}=m_{i} / \delta t$ and $\tilde{b}=b / \delta t^{2}$. Since the above fraction will grow infinitely large for $\delta t \rightarrow 0$ the instability of the simulation is not surprising. If the $3 \mathrm{D}$ artery is rigid however, then the capacitance $C$ is 0 and consequently $a_{1}=b=0$. Then the fraction tends to 0 as $\delta t \rightarrow 0$ and the method is stable for small enough $\delta t$. Indeed, without fluid-structure interaction the simulation is stable (figure 4).

A solution for the simulation with fluid-structure interaction would be to reverse the iteration order but then an additional problem arises. Currently, the pressure is used as input for the 3D elastic artery and can simply be assumed constant over the cross-section. However, when using the velocity as input, the flux obtained from the electric network needs to be complemented with a velocity profile (Poiseuille, Womersley) to bridge the transition from OD to 3D. Therefore we choose to use the quasi-simultaneous method instead.

For use in the quasi-simultaneous method we approximate the $3 \mathrm{D}$ elastic artery with an RLC circuit. Then every time step part of the 3D CFD model (the RLC approximation) is solved simultaneously with the circulation model while the remaining part is treated in a weak manner. The method is successful in stabilizing the system, as can be seen in figure 4 , where $q_{\text {in }}$ and $q_{\text {out }}$ are plotted, alongside the rigid solution. The effect of elasticity on flow parameters is clearly visible; the flow is significantly increased.

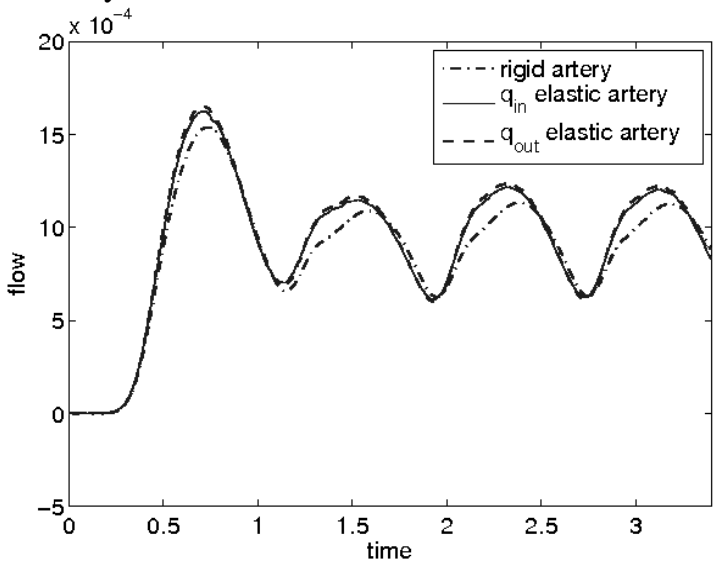

Fig. 4 Pulsatile flow through the artery of the system in figure 1 


\section{B. Fluid-structure interaction}

Based on a simplified model, a surprisingly accurate (in)stability condition can be derived for fluid-structure interaction in an elastic artery. For the wall dynamics the independent rings model [4] can be used (here with Poisson ratio $v=0): \rho_{s} h \eta_{t t}+\left(E h / r_{0}^{2}\right) \eta=p$, where $\rho_{s}$ is the density of the structure (vessel wall), $E$ Young's modulus, $h$ the wall thickness, $p$ the transmural pressure and $\eta(x, t)=r(x, t)-r_{0}$ the radial displacement of the vessel wall. For the fluid dynamics the following highly simplified equation is obtained from the $1 \mathrm{D}$ equations for conservation of mass and momentum in a tube: $2 r_{t t} / r_{0}=p_{x x} / \rho_{f}$, with $\rho_{f}$ the density of the fluid. This equation can also be used to estimate pulse wave velocity [4]. The fluid and wall equations are similar in form to (1), except for the spatial derivative $p_{x x}$. This is resolved by considering Fourier modes. Then the stability condition simply follows from (5):

$$
\rho_{s} h>\frac{2 l^{2} \rho_{f}}{\pi^{2} r_{0}} \pm \frac{\partial t^{2}}{4} \frac{E h}{r_{0}^{2}},
$$

where $l$ is the length of the tube. The \pm sign refers to the explicit or implicit discretization of the elastic term in the independent rings model. Here it is assumed that the pressure is used as input for the wall model and wall acceleration is used as input for the fluid model. An alternative derivation of the first part of (9) is given in [5].

The explicit and implicit theoretical stability bounds (9) are plotted in figure 5 with the mass and stiffness coefficients of the independent rings model on the axes. These bounds are separating the theoretically stable upper region from the unstable lower region. To get an idea of the quality of the theoretical conditions, dynamic (Womersley) 3D fluid-structure interaction simulations are performed for various values of the wall stiffness and increased wall mass to look for critical points, where the simulation is just about stable. These critical points are the circular markers in figure 5. As can be seen, the theoretical bound based on the simplified model does a very good job of predicting the performance of the full model.


Fig. 5 Theoretical and experimental stability comparison

The mass of the arterial wall is too small for condition (9) to be valid (at least for the larger arteries). Therefore, in order to obtain a stable fluid-structure interaction algorithm, we apply the quasi-simultaneous method, with the independent rings model as interaction law. This allows more complex wall models to be coupled in a stable manner.

\section{Conclusions}

When weak coupling methods yield unstable results it is not always necessary to turn to strong coupling methods: if suitable approximations of submodels are available the quasi-simultaneous method is a good alternative. Then instead of interweaving a complex set of subsystems only simple submodels need to be integrated. The results are promising: at low computational cost, stability is achieved where weak methods fail.

\section{REFERENCES}

1. Ottesen JT, Olufsen MS, Larsen JK (2004) Applied mathematical models in human physiology, SIAM, Monographs on Mathematical Modeling and Computation.

2. Maurits NM, Loots GE, Veldman AEP (2007) The influence of vessel wall elasticity and peripheral resistance on the flow wave form: a CFD model compared to in-vivo ultrasound measurements. J. Biomech. 40(2):427-436.

3. Loots E, Hillen B, Veldman AEP (2003) The role of hemodynamics in the development of the outflow tract of the heart. J. Eng. Math 45:91-104.

4. Fung YC (1997) Biomechanics, circulation. 2nd Edition, Springer.

5. Causin P, Gerbeau JF, Nobile F (2004) Added-mass effect in the design of partitioned algorithms for fluid-structure interaction. Comput. Methods Appl. Mech. Engrg. 194:4506-4527.

$\begin{array}{lll}\text { - } & \text { Author: } & \text { G. Rozema } \\ \text { - } & \text { Institute: } & \text { University of Groningen } \\ \text { - } & \text { Street: } & \text { Nijenborgh } 9 \\ \text { - } & \text { City: } & \text { Groningen } \\ \text { - } & \text { Country: } & \text { the Netherlands } \\ \text { - } & \text { Email: } & \text { g.rozema@ math.rug.nl }\end{array}$

